Supplemental Information

\title{
Evaluating Combined Precursor Isotopic Labeling and Isobaric Tagging Performance on Orbitraps To Study the Peripheral Proteome of Alzheimer's Disease
}

\author{
Christina D. King ${ }^{1}$ and Renã A.S. Robinson ${ }^{1-5 *}$ \\ ${ }^{1}$ Department of Chemistry, Vanderbilt University, Nashville, Tennessee 37235, United States \\ ${ }^{2}$ Department of Neurology, Vanderbilt University Medical Center, Nashville, Tennessee 37232, United States \\ ${ }^{3}$ Vanderbilt Memory \& Alzheimer's Center, Vanderbilt University Medical Center, Nashville, Tennessee 37212, United States \\ ${ }^{4}$ Vanderbilt Institute of Chemical Biology, Vanderbilt University, Nashville, Tennessee 37232, United States \\ ${ }^{5}$ Vanderbilt Brain Institute, Vanderbilt University, Nashville, Tennessee 37232, United States \\ * Author to whom correspondence should be addressed: \\ Prof. Renã A. S. Robinson \\ Department of Chemistry \\ Vanderbilt University \\ 5423 Stevenson Center
}

Nashville, TN 37235

Tel: $1-615-343-0129$

Email address: rena.as.robinson@vanderbilt.edu 
Supplemental Information

Supplemental Methods

Supplemental Figures

Supplemental Figure S1: Comparison of proteins quantified in fractionated datasets analyzed on the Fusion Lumos and the Orbitrap Velos and proteins quantified in all tissues.

Supplemental Figure S2: Comparison of a) protein and b) peptide identifications, and c) quantified spectra with gradient times of 105, 120, and $150 \mathrm{~min}$.

Supplemental Figure S3: Effects of dynamic exclusion times 0, 10, and 20 s on protein identifications of top) light and bottom) heavy dimethylated peptides.

Supplemental Figure S4: Distribution of PSMs detected among identified proteins.

Supplemental Figure S5: Canonical pathways of statistically-significant proteins in top) brain and bottom) heart tissues.

Supplemental Figure S6: Canonical pathways of statistically-significant proteins in liver tissues.

Supplemental Figure S7: Western Blot analyses of differentially - expressed proteins in the brain, heart, or liver as function of disease.

$\underline{\text { Supplemental Tables }}$

Supplemental Table S1: Strategy of cPILOT analysis across tissues.

Supplemental Table S2: Effects of targeted analyses tests on the number of PSMs quantified.

Supplemental Table S3: Effects of targeted analyses tests on the number of proteins quantified.

Supplemental Table S4: Peptides identified from brain, heart, and/or liver tissues (batches 1 3, combined experiments, Excel file).

Supplemental Table S5: Protein groups identified from brain, heart, and/or liver tissues (batches $1-3$, combined experiments, Excel file)

Supplemental Table S6: Proteins identified and quantified across batches in each experiment.

Supplemental Tables S7a-c: Proteins identified and quantified across tissue/disease-state.

Supplemental Table S8: Proteins quantified in one or two tissue types.

Supplemental Table S9: Differentially - expressed proteins in the brain, heart, and/or liver as a function of disease. 


\section{Supplemental Methods}

Liquid Chromatography gradients used:

a) 0 - 10 min, $10 \%$ mobile phase $\mathrm{B} ; 10-67 \min , 10-30 \% \mathrm{~B} ; 67-75 \min , 30-60 \% \mathrm{~B} ; 75-$ 77 min, 60 - 90\% B; $77-82 \min , 90 \%$ B; $82-83 \min , 90-10 \%$ B; $83-105 \min , 10 \%$ B.

b) 0 - 10 min, $10 \%$ mobile phase $\mathrm{B} ; 10-30 \mathrm{~min}, 10-15 \% \mathrm{~B} ; 30-75 \mathrm{~min}, 15-30 \% \mathrm{~B} 75-$ 88 min, $30-60 \%$ B; 88 - 92 min, $60-90 \%$ B; $92-99 \min , 90 \%$ B; $99-100$ min, $90-10 \%$ B; $100-120 \mathrm{~min}, 10 \% \mathrm{~B}$.

c) 0 - 10 min, $10 \%$ mobile phase $\mathrm{B} ; 10-30 \mathrm{~min}, 10-15 \% \mathrm{~B} ; 30-100 \mathrm{~min}, 15-30 \% \mathrm{~B} 100$ - $118 \min , 30-60 \% \mathrm{~B} ; 118-123 \mathrm{~min}, 60-90 \% \mathrm{~B} ; 123-129 \min , 90 \% \mathrm{~B} ; 129-130 \mathrm{~min}, 90-$ $10 \% \mathrm{~B} ; 130-150 \mathrm{~min}, 10 \% \mathrm{~B}$.

Elite: 0 - 7 min, $10 \%$ mobile phase B; $7-27 \min , 10-15 \% \mathrm{~B} ; 27-102 \mathrm{~min}, 15-20 \% \mathrm{~B} ; 102-$ 122 min, $20-30 \%$ B; $122-132 \min , 30-60 \%$ B; $133-137$ min, $60-80 \%$ B; $137-150$ min, $80 \% \mathrm{~B}, 150-180,10 \% \mathrm{~B}$.

\section{Mass Spectrometry analyses:}

Peptides were analyzed using three methods (analyses methods $1-3$ ). (1) Online desalting and reversed-phase chromatography was performed with a nano-UHPLC system equipped with an autosampler (Dionex, ThermoFisher Scientific). Mobile phases A and B used for separation were (v/v) $0.1 \%$ FA and $100 \%$ ACN with $0.1 \%$ FA, respectively. Peptides ( $250 \mathrm{ng}$ ) were loaded onto a commercial (Thermo Fisher Scientific) trapping column $(75 \mu \mathrm{m} \times 2 \mathrm{~cm})$ containing $\mathrm{C}_{18}(3 \mu \mathrm{m}$, $100 \AA$ ) at $2 \mu \mathrm{L}^{*} \mathrm{~min}^{-1}$ in $0.1 \%$ FA for $10 \mathrm{~min}$. After desalting, the sample was loaded onto an analytical column $(100 \mu \mathrm{m}$ i.d. x $23 \mathrm{~cm})$, which was packed in-house with $\mathrm{C}_{18}(2.5 \mu \mathrm{m}, 150 \AA$, Waters). Four gradients were used to separate peptides.

Standard data-dependent acquisition parameters were as follows: the MS survey scan in the Orbitrap $(375-1500 \mathrm{~m} / \mathrm{z})$ was 120,000 resolution; the most intense peaks with $3 \mathrm{~s}$ (Top Speed) were isolated $(0.7 \mathrm{~m} / \mathrm{z})$ and fragmented with collision-induced dissociation (CID) in the ion trap with an NCE of 35\%, AGC of $1 \times 10^{4}$, dynamic exclusion of $20 \mathrm{~s}$, ppm mass tolerance of 10 , maximum IT of $100 \mathrm{~ms}$. Directly after each MS/MS scan, the four most intense fragment ions (over varying $\mathrm{m} / \mathrm{z}$ ranges) were selected for an additional fragmentation (i.e. $\mathrm{MS}^{3}$ ) event by HCD and analyzed in the OT (scan range: $100-400 \mathrm{~m} / z$, isolation width: $2 \mathrm{~m} / z$, AGC: $5 \times 10^{4}$, NCE: 55\%, resolution: 60,000, maximum IT: $118 \mathrm{~ms}$ ). Other parameters such as precursor selection range, precursor ion exclusion, and isobaric tag loss exclusion were set as default. Parameters including precursor isolation width, dynamic exclusion, targeted analyses, and SPS selection were tested and varied. Each sample was subject to triplicate injections.

(2) Peptides were loaded onto the same commercial trap column as (1). Separation occurred on the same in-house analytical column, but the gradient was adjusted to 120 min. Adjustments made for Full MS acquisition included widening the precursor isolation window to $2 \mathrm{~m} / \mathrm{z}$ and using the targeted mass difference node. To obtain reporter ion signal, the four most intense 
fragment ions were increased to ten (over varying $\mathrm{m} / \mathrm{z}$ ranges) for an additional fragmentation event (i.e. $\mathrm{MS}^{3}$ ) event by HCD and analyzed in the OT. Each sample was subject to duplicate injections.

(3) Online desalting and reversed-phase chromatography was performed with a nano-HPLC system equipped with an autosampler (Eksigent) as previously described. SCX fractions $(6 \mu \mathrm{L})$ were loaded onto a trapping column $(100 \mu \mathrm{m}$ i.d. x $2 \mathrm{~cm})$, at $3 \mu \mathrm{L} / \mathrm{min}$ in $3 \%$ mobile phase B for 3 min. After desalting, the sample was loaded onto an analytical column $(75 \mu \mathrm{m}$ i.d. $x 13.2 \mathrm{~cm})$. The LC eluent was analyzed with positive mode nanoflow electrospray using a LTQ Orbitrap Velos mass spectrometer (Thermo Fisher Scientific). Briefly, DDA parameters were as follows: the MS survey scan in the Orbitrap $(300-1800 \mathrm{~m} / \mathrm{z}$ ) was 60,000 resolution; the top $1-7$ and top $8-14$ most intense peaks were isolated and fragmented with collision-induced dissociation (CID) in the LTQ with an NCE of 35\%. Directly after each MS/MS scan, the most intense fragment ion (over $200-1545 \mathrm{~m} / \mathrm{z}$ ) was selected for an additional fragmentation (i.e. $\mathrm{MS}^{3}$ ) by HCD (isolation width: $4 \mathrm{~m} / z$, AGC: $3 \times 10^{5}$, NCE: 60\%, resolution: 7500, maximum IT: $250 \mathrm{~ms}$ ). Each fraction was subject to triplicate injections.

\section{$\underline{\text { Targeted analyses parameters used in MS analyses }}$}

Targeted inclusion and exclusion tests were performed using the following nodes: targeted mass, targeted mass difference, targeted isotopic ratio, and targeted mass trigger. In both targeted mass, and targeted mass trigger nodes, a list including $m / z, \mathrm{z}, \mathrm{m}$, and retention time (targeted mass only) are imported into a list. In targeted mass difference, two precursors were listed for the targeted group. In addition, a mass difference of 8.0444 Da (Heavy DM - Light DM) and 7.0381 Da (Dimethyl 7-Light DM) were listed, the partner intensity range relative to the most intense precursor was set to $70-100 \%$, a subsequent scan was performed on both ions in the pair and the charge state for ions in the pair had to be the same. For targeted isotopic ratio, similar parameters were added, with the distinction that the predicted isotopic range was set to $0.7-1$.

\section{Data analysis}

Raw files were analyzed with Proteome Discoverer v. 2.1 and 2.2 software (Thermo Scientific). Spectra were used to obtain sequence information against the Uniprot M. musculus database (01/19/2018, 53035 sequences). SEQUEST HT search parameters were as follows: two maximum trypsin miscleavages, precursor mass tolerance of $15 \mathrm{ppm}(\mathrm{OT})$, fragment mass tolerance of $1 \mathrm{Da}$ (IT); static modifications were either light or heavy/dimethyl 7Da Dimethyl $/+28.031$ or 36.028/35.070 Da (N-terminus) and carbamidomethyl modification/+57.021 Da (Cys); dynamic modifications were TMT six-plex/+229.163 Da (Lys) and oxidation modification/+15.995 Da (Met). Decoy database searching was employed to generate medium (false discovery rate, FDR, of 5\% for all identifications) confidence peptide lists. All peptides with medium confidence were used to identify and quantify proteins. To filter peptides, the following parameters were applied: peptides with a peptide to spectral match (PSM) $>1$ across biological cohorts, peptide confidence level of medium, peptide rank of 1 , peptide deviation of $10 \mathrm{ppm}$, and $\mathrm{S} / \mathrm{N} \geq 10$. The reporter ions (i.e. $m / z 126-131$ ) were identified with the following parameters: most confident centroid and 
$30 \mathrm{ppm}$ for reporter ion mass tolerance. Proteins quantified in 5 or 6 biological replicates (per tissue type) were selected for data normalization.

Data verification by SDS-PAGE and Western Blot

Brain, heart, and liver tissues from AD and WT mice were used for verification by Western blot. Protein was fractionated via SDS Page ( $120 \mathrm{~V}$ loading, $160 \mathrm{~V}$ for $\sim 70 \mathrm{~min}$ ). Proteins were transferred to a nitrocellulose membrane using a wet transfer at $100 \mathrm{~V}$ for $70 \mathrm{~min}$. After incubation with 5\% nonfat milk in TBST (Tris-buffered saline with Tween-20; $50 \mathrm{mM}$ Tris, 150 $\mathrm{mM} \mathrm{NaCl}$, adjusted to $\mathrm{pH} \mathrm{7.4,0.1 \%} \mathrm{Tween-} 20$ overnight, the membranes were washed $4 \times 4$ min with TBST and incubated overnight with antibodies against $\beta$-actin (proteintech 20536-1-AP; 1:1000) or PSMD11 (proteintech 14786-1-AP; 1:1000) at $4{ }^{\circ} \mathrm{C}$. Membranes were washed $4 \times 4$ min with TBST and incubated with a 1:10,000 dilution of fluorescent-labeled anti-mouse (Starbright Blue 700; Bio-Rad Laboratories) for $60 \mathrm{~min}$. Blots were washed $4 \times 4$ min with TBST prior to imaging using a ChemiDoc MP imaging system (Bio-Rad). ImageLab software (Bio-Rad version 6.0) was used for band quantification. 


\section{Supplemental Figures}

a)

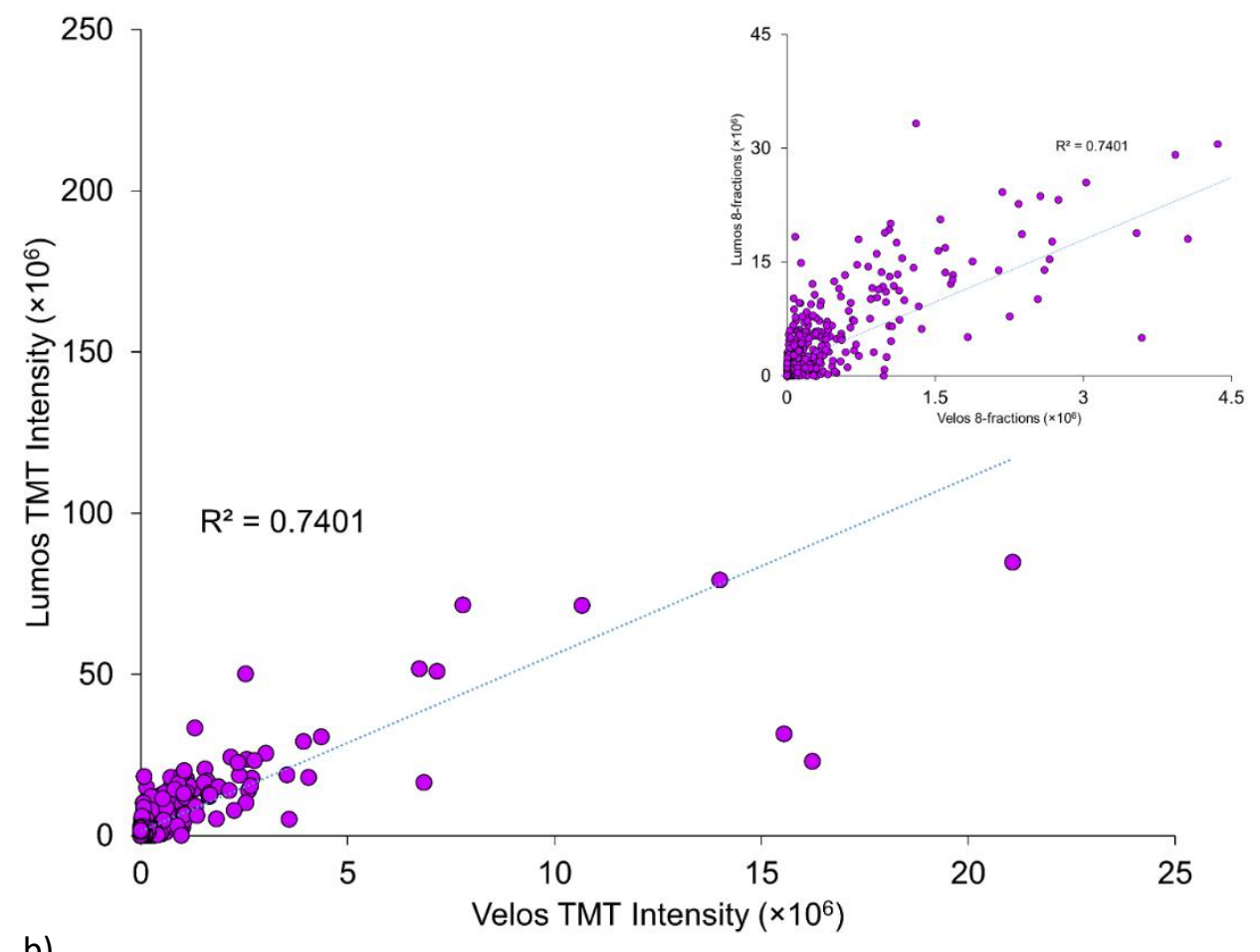

b)

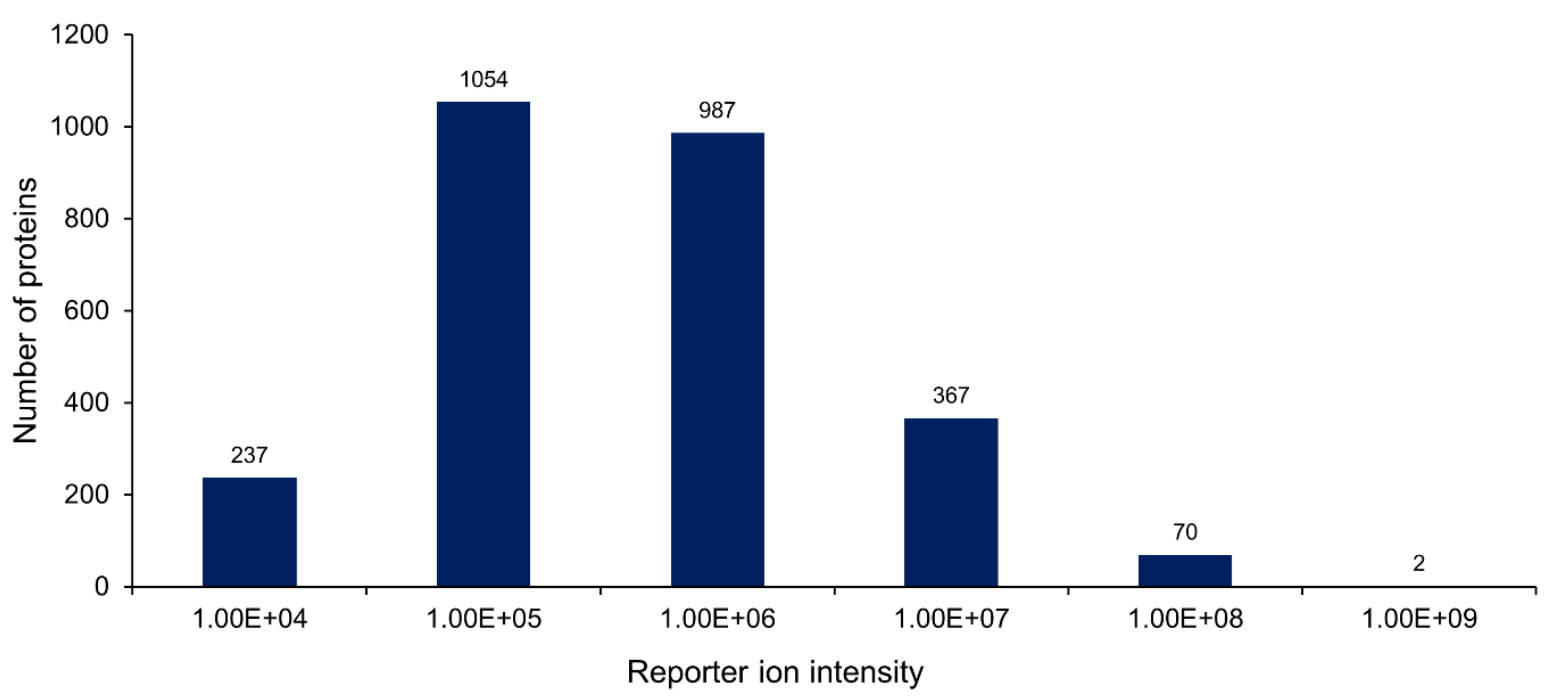

Supplemental Figure S1: a) Comparison of proteins quantified in fractionated datasets analyzed on the Fusion Lumos and the Orbitrap Velos and b) reporter ion intensities of proteins quantified across brain, heart, and liver tissues (Figure 4a). Reporter ion ratios of proteins quantified in both Fusion Lumos and Orbitrap Velos datasets were compared for differences across signal. 
$105 \mathrm{~min}$

$120 \mathrm{~min}$

$150 \mathrm{~min}$

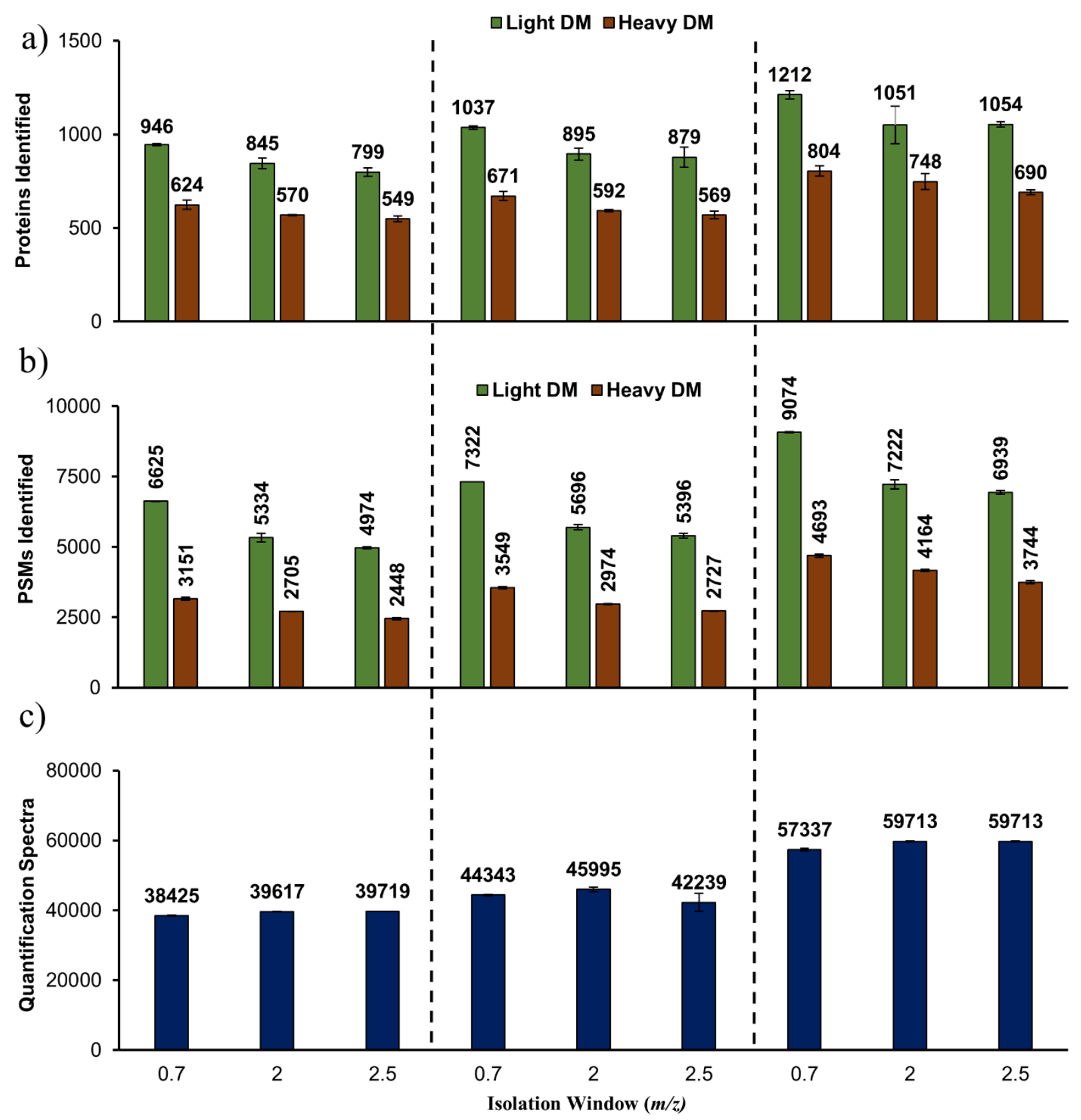

Supplemental Figure S2: Comparison of a) protein and b) peptide identifications, and c) quantified spectra with gradient times of 105, 120, and $150 \mathrm{~min}$. 


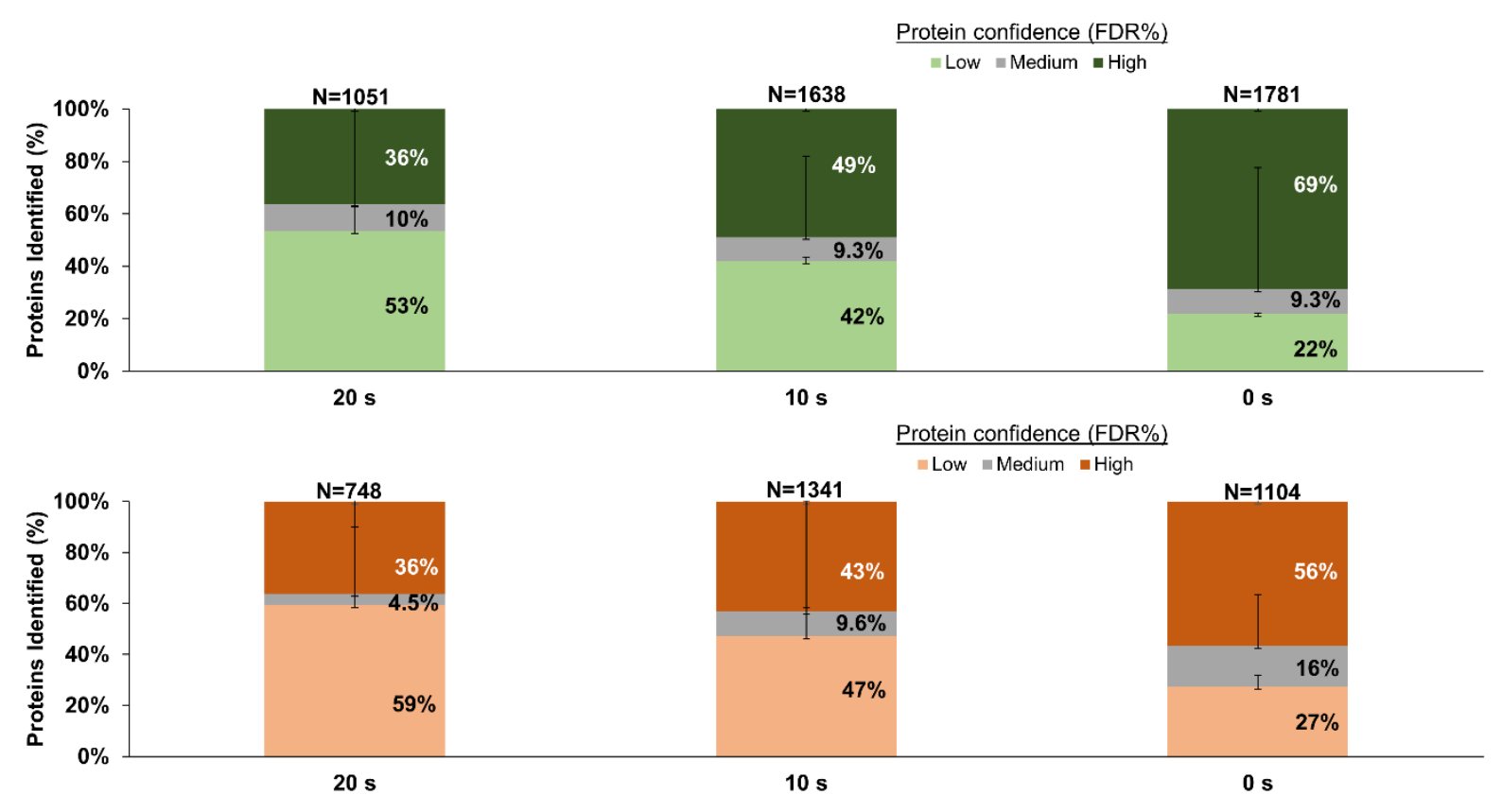

Supplemental Figure S3: Effects of dynamic exclusion times 0, 10, and $20 \mathrm{~s}$ on protein identifications of top) light and bottom) heavy dimethylated peptides. Proteins identified ( $\mathrm{N}=1638$ light, $\mathrm{N}=1341$, heavy) had false discovery rates (FDR) of $<1 \%$ (high), $<5 \%$ (medium), or $>5 \%$ (low). 


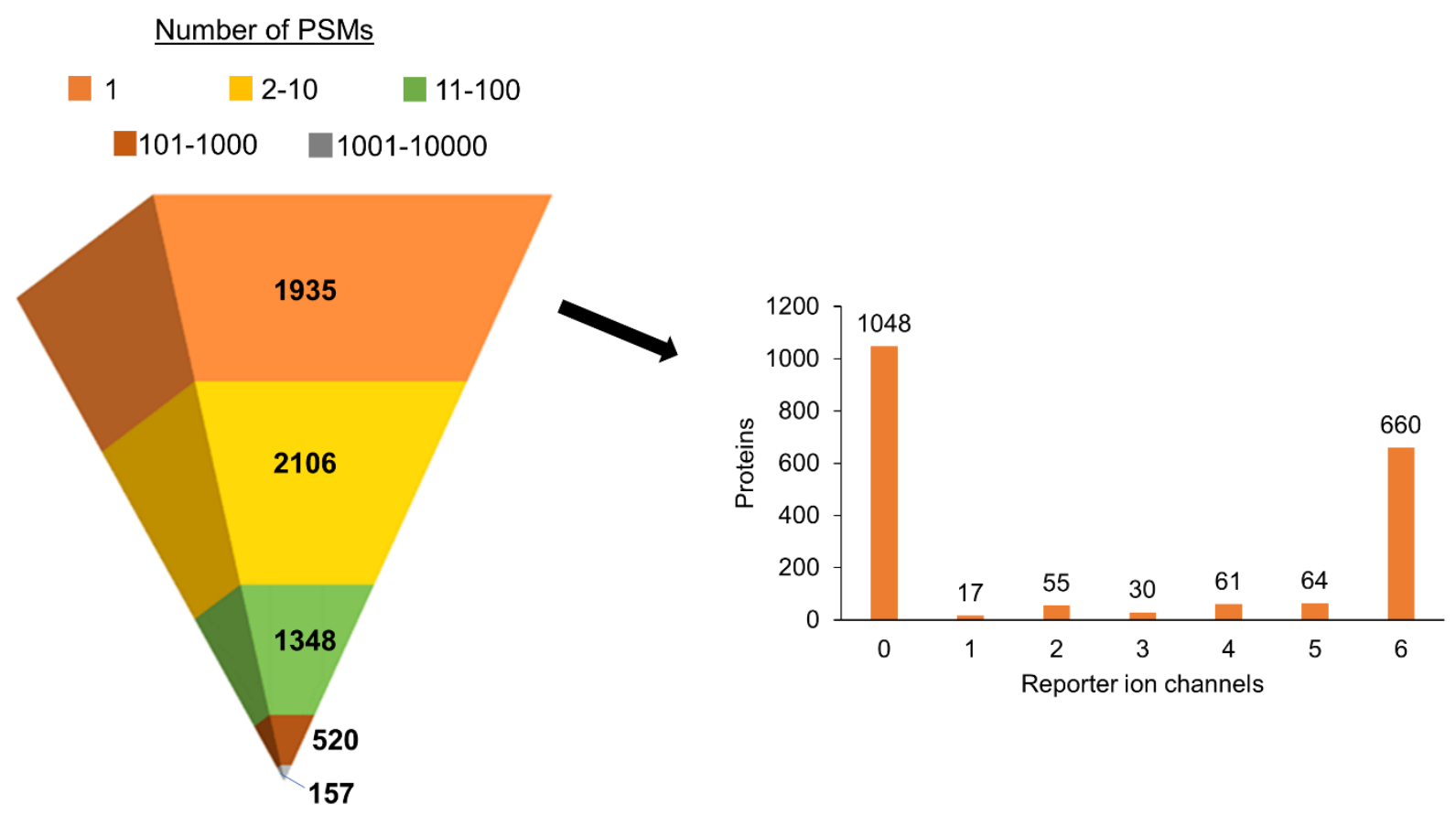

Supplemental Figure S4: Distribution of PSMs detected among identified proteins. Proteins were identified from a range of 1 - > 10000 PSMs (see legend) per protein. Zoomed-in views of proteins identified from 1 PSM (right) are quantified from a range of $0-6$ reporter ion channels which may correspond from $0-3$ tissues. 

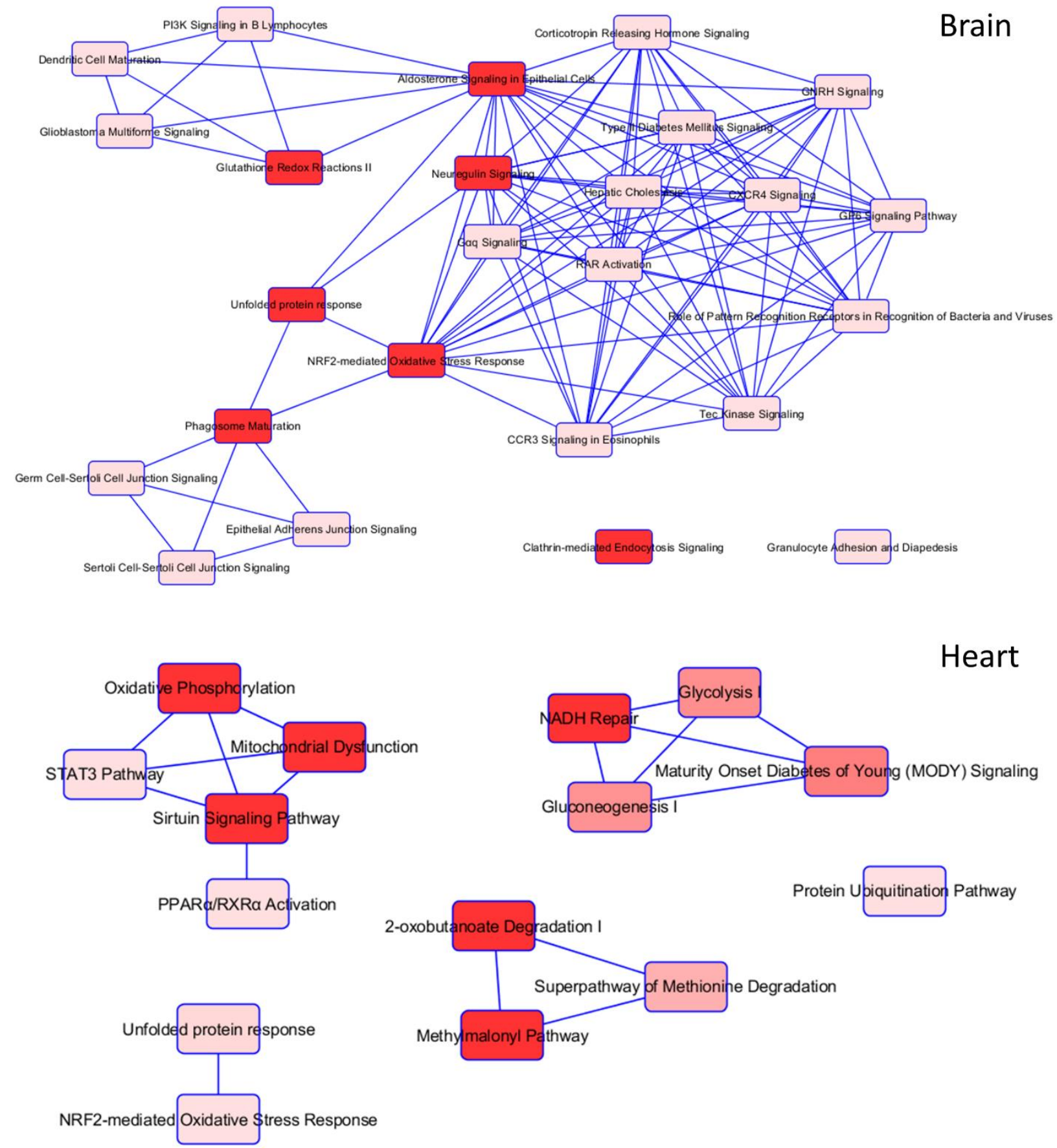

Supplemental Figure S5: Canonical pathways of differentially - expressed proteins in brain and heart, liver tissues as a function of disease ( $\left.\mathrm{AD} / \mathrm{WT}_{\text {tissue }}\right)$. 


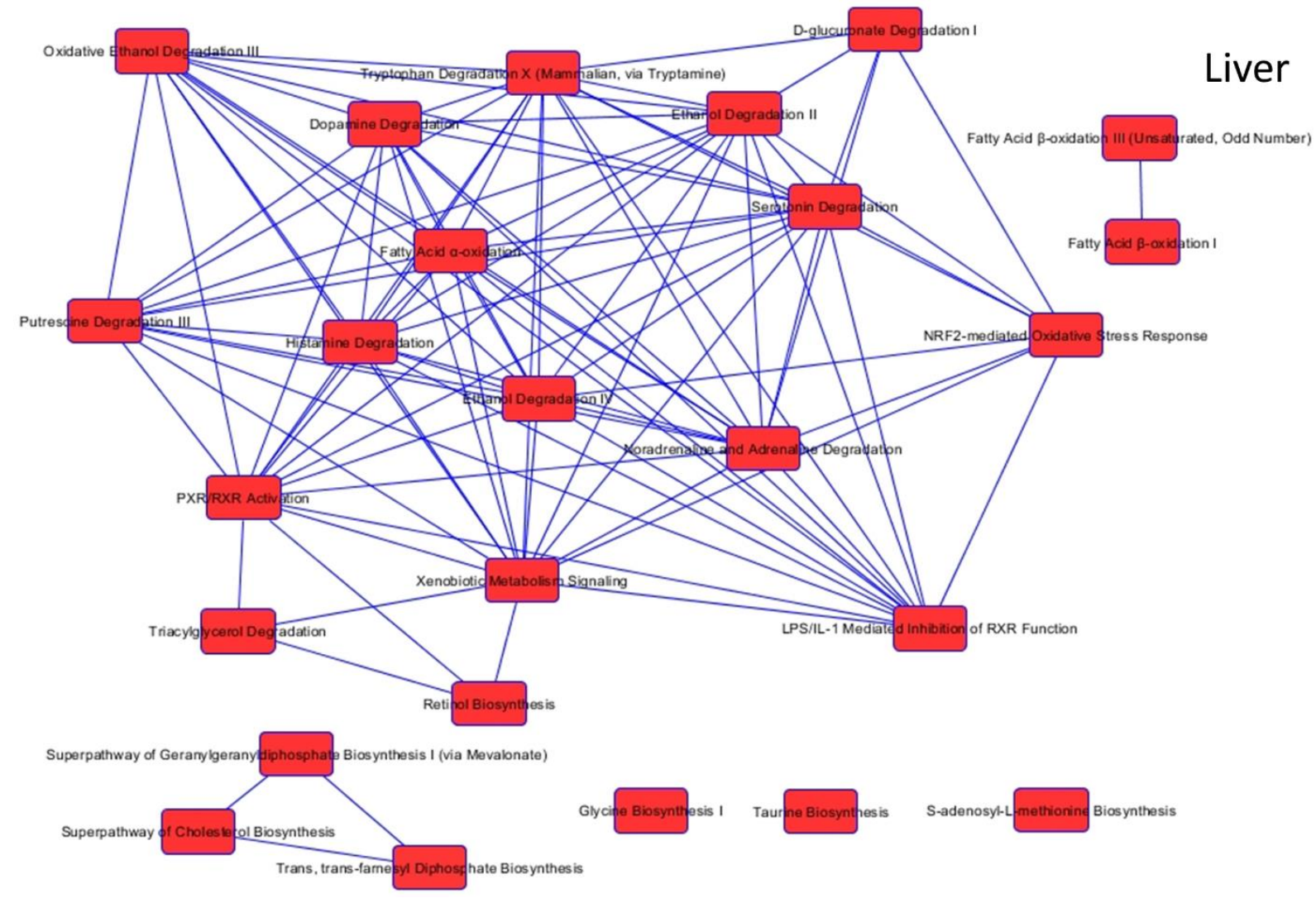

Supplemental Figure S6: Canonical pathways of differentially - expressed proteins in liver tissues as a function of disease ( $\left.\mathrm{AD} / \mathrm{WT}_{\text {tissue }}\right)$. 
a)

\section{Beta actin 1}

AD WT AD WT AD WT

liver liver heart heart brain brain
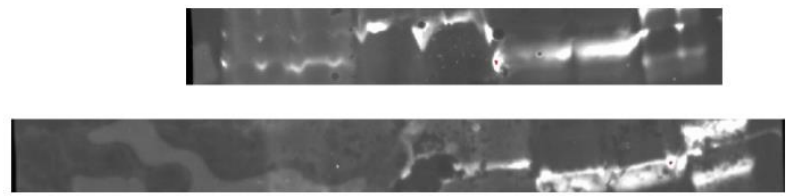

b) PSMD11

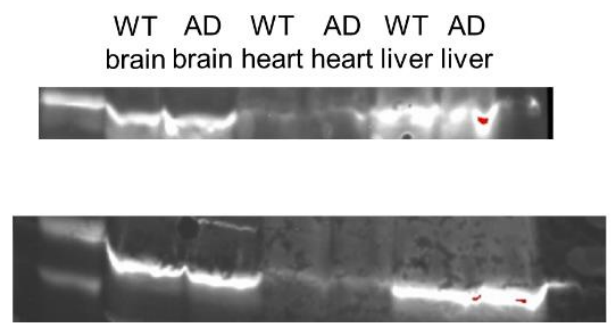

Supplemental Figure S7: Western Blot analyses of differentially - expressed proteins in the brain, heart, or liver as function of disease. a) Beta actin (control) and b) PSMD11 were probed in WT and AD brain, heart, and liver tissues. 


\section{Supplemental Tables}

Supplemental Table S1: Strategy of cPILOT analysis across tissues.

\begin{tabular}{lcccccc}
\hline TMT $^{6}$-plex tag & $\mathbf{1 2 6}(\mathbf{W T})$ & $\mathbf{1 2 7}(\mathbf{A D})$ & $\mathbf{1 2 8}(\mathbf{W T})$ & $\mathbf{1 2 9}(\mathbf{A D})$ & $\mathbf{1 3 0}(\mathbf{W T})$ & $\mathbf{1 3 1}$ (AD) \\
\hline Light DM-Batch 1 & Brain & Brain & Heart & Heart & Liver & Liver \\
Heavy DM-Batch 1 & Heart & Heart & Liver & Liver & Brain & Brain \\
Light DM-Batch 2 & Liver & Liver & Heart & Heart & Brain & Brain \\
Heavy DM-Batch 2 & Brain & Brain & Liver & Liver & Heart & Heart \\
Light DM-Batch 3 & Heart & Heart & Brain & Brain & Liver & Liver \\
Heavy DM-Batch 3 & Heart & Heart & Brain & Brain & Liver & Liver \\
\hline
\end{tabular}

"Light DM" and "Heavy DM" samples are dimethylated by $-\mathrm{CH}_{3} \mathrm{CH}_{3}$ or $-{ }^{13} \mathrm{C}^{2} \mathrm{H}_{3}{ }^{13} \mathrm{C}^{2} \mathrm{H}_{3}$, respectively. 
Supplemental Table S2: Effects of targeted analyses tests on the number of PSMs quantified.

\begin{tabular}{|c|c|c|c|c|c|c|c|c|}
\hline \multirow[t]{2}{*}{ Targeted Test } & \multicolumn{2}{|c|}{ PSMs ID } & \multicolumn{2}{|c|}{$\mathbf{R}(\%)^{\mathbf{a}}$} & \multicolumn{2}{|c|}{$\mathbf{K}(\%)^{\mathbf{b}}$} & \multicolumn{2}{|c|}{ TMT-K $(\%)^{\mathrm{c}}$} \\
\hline & Light & Heavy & Light & Heavy & Light & Heavy & Light & Heavy \\
\hline Targeted Mass & & & & & & & & \\
\hline Trigger & $7378 \pm 204$ & $4265 \pm 21$ & 3159 (42.8) & $1725(40.4)$ & $4079(55.3)$ & $2440(57.2)$ & 3985 (97.7) & 2375 \\
\hline Targeted Mass & & & & & & & & \\
\hline Difference & $7403 \pm 62$ & $8059 \pm 53$ & $3172(42.8)$ & $3454(42.8)$ & $4048(54.7)$ & $4384(54.4)$ & 3957 (97.7) & $4263(97.2)$ \\
\hline $\begin{array}{l}\text { Targeted } \\
\text { Isotopic Ratio }\end{array}$ & $7078 \pm 25$ & $3890 \pm 45$ & $3061(43.2)$ & $1577(43.2)$ & $3878(54.8)$ & $2228(54.8)$ & 3795 (97.9) & 2169 \\
\hline $\begin{array}{l}\text { Targeted Mass } \\
\text { (Exclusion) }\end{array}$ & $6599 \pm 117$ & $3498 \pm 25$ & $2536(38.4)$ & 1199 (34.3) & $3934(59.6)$ & 2197 (59.6) & 3854 (98.0) & $2146(97.7)$ \\
\hline $\begin{array}{l}\text { Targeted Mass } \\
\text { (Inclusion) }\end{array}$ & $1744 \pm 883$ & $1941 \pm 963$ & $47(2.7)$ & $113(5.8)$ & 1695 (97.2) & $1825(94.0)$ & 1676 (98.9) & $1810(99.2)$ \\
\hline
\end{tabular}


Supplemental Table S3: Effects of targeted analyses tests on the number of proteins quantified

\begin{tabular}{|c|c|c|c|c|c|c|}
\hline \multirow[t]{2}{*}{ Targeted Test } & \multicolumn{2}{|c|}{ Protein Groups } & \multicolumn{2}{|c|}{ Proteins $^{\mathrm{a}}$ (1 Channel) } & \multicolumn{2}{|c|}{ Proteins $^{b}$ (6 Channels) } \\
\hline & Light & Heavy & Light & Heavy & Light & Heavy \\
\hline Targeted Mass Trigger & $1074 \pm 11$ & $766 \pm 11$ & $764 \pm 4$ & $535 \pm 10$ & $716 \pm 2$ & $503 \pm 3$ \\
\hline Targeted Mass Difference & $1106 \pm 6$ & $973 \pm 26$ & $774 \pm 18$ & $655 \pm 15$ & $704 \pm 16$ & $606 \pm 21$ \\
\hline Targeted Isotopic Ratio & $1086 \pm 13$ & $727 \pm 38$ & $756 \pm 16$ & $523 \pm 33$ & $701 \pm 9$ & $490 \pm 28$ \\
\hline $\begin{array}{l}\text { Targeted Mass } \\
\text { (Exclusion) }\end{array}$ & $1002 \pm 40$ & $660 \pm 14$ & $726 \pm 30$ & $497 \pm 8$ & $680 \pm 18$ & $458 \pm 3$ \\
\hline Targeted Mass (Inclusion) & $614 \pm 294$ & $523 \pm 256$ & $524 \pm 263$ & $452 \pm 229$ & $471 \pm 259$ & $407 \pm 228$ \\
\hline
\end{tabular}


Supplemental Table S4: Peptides identified from brain, heart, and/or liver tissues (batches 1-3, combined experiments).

-see supplemental tables Excel file 
Supplemental Table S5: Protein groups identified from brain, heart, and/or liver tissues (batches 1-3, combined experiments).

-see supplemental tables Excel file 
Supplemental Table S6: Proteins identified and quantified across batches in each experiment.

\begin{tabular}{|c|c|c|c|}
\hline & 8 Fractions (Velos) & No Fractions (Lumos) & 8 Fractions (Lumos) \\
\hline${ }^{\mathrm{b}}$ Batch 1 & 758 & 783 & 2421 \\
\hline${ }^{\mathrm{b}}$ Batch 2 & 1029 & 910 & 2396 \\
\hline${ }^{\mathrm{b}}$ Batch 3 & 830 & 864 & 2561 \\
\hline${ }^{\mathrm{c}}$ Average \pm SD & $872 \pm 140$ & $852 \pm 64$ & $2459 \pm 89$ \\
\hline
\end{tabular}

aThe number of proteins identified across three batches; 8 Fractions Velos ( $N=2199)$, No Fractions Lumos ( $N=1848)$, and 8 Fractions Lumos $(\mathrm{N}=4968) .{ }^{\mathrm{b}}$ The number of proteins quantified per batch run. ${ }^{\mathrm{C}}$ The average number of proteins quantified across 3 batches. 
Supplemental Tables S7a-c: Proteins identified and quantified across tissue/disease-state.

\begin{tabular}{|c|c|c|c|c|c|c|}
\hline \multicolumn{7}{|c|}{8 Fractions (Velos) } \\
\hline & WT Brain & AD Brain & WT Heart & AD Heart & WT Liver & AD Liver \\
\hline bBiological Replicate 1 & 496 & 523 & 491 & 503 & 529 & 527 \\
\hline cBiological Replicate 2 & 393 & 391 & 352 & 370 & 388 & 392 \\
\hline 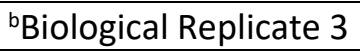 & 709 & 581 & 726 & 719 & 757 & 742 \\
\hline 'Biological Replicate 4 & 553 & 552 & 500 & 428 & 558 & 555 \\
\hline biological Replicate 5 & 453 & 466 & 404 & 412 & 501 & 500 \\
\hline 'Biological Replicate 6 & 390 & 397 & 351 & 345 & 413 & 406 \\
\hline dAverage \pm SD & ${ }^{b} 499 \pm 120$ & $485 \pm 80$ & $471 \pm 141$ & $463 \pm 137$ & $524 \pm 132$ & $520 \pm 127$ \\
\hline
\end{tabular}

The number of proteins identified in each tissue/disease-state ( $N$ ranges between 1-6 biological replicates); WT Brain ( $N=1023), A D$ Brain ( $N=959)$, WT Heart ( $N=1017)$, AD Heart ( $N=978)$, WT Liver ( $N=1052)$, and AD Liver ( $N=1048)$.

\begin{tabular}{|c|c|c|c|c|c|c|}
\hline \multicolumn{7}{|c|}{ No Fractions (Lumos) } \\
\hline & WT Brain & AD Brain & WT Heart & AD Heart & WT Liver & AD Liver \\
\hline${ }^{\text {bBiological Replicate 1 }}$ & 658 & 656 & 658 & 658 & 658 & 657 \\
\hline${ }^{\text {cBiological Replicate 2 }}$ & 573 & 570 & 574 & 574 & 574 & 574 \\
\hline${ }^{\text {bBiological Replicate 3 }}$ & 788 & 785 & 784 & 785 & 783 & 783 \\
\hline${ }^{\text {BBiological Replicate 4 }}$ & 640 & 640 & 639 & 636 & 639 & 640 \\
\hline${ }^{\text {bBiological Replicate 5 }}$ & 732 & 735 & 731 & 731 & 732 & 734 \\
\hline${ }^{\text {cBiological Replicate 6 }}$ & 620 & 621 & 616 & 617 & 615 & 619 \\
\hline${ }^{\mathrm{d}}$ Average \pm SD & $669 \pm 78$ & $668 \pm 79$ & $667 \pm 77$ & $667 \pm 78$ & $667 \pm 77$ & $668 \pm 77$ \\
\hline
\end{tabular}

aThe number of proteins identified in each tissue/disease-state ( $N$ ranges between 1-6 biological replicates); WT Brain ( $N=1283$ ), AD Brain $(\mathrm{N}=1279)$, WT Heart $(\mathrm{N}=1279)$, AD Heart $(\mathrm{N}=1278)$, WT Liver $(\mathrm{N}=1278)$, and AD Liver ( $\mathrm{N}=1280)$.

\begin{tabular}{|c|c|c|c|c|c|c|}
\hline \multicolumn{7}{|c|}{ 8 Fractions (Lumos) } \\
\hline & WT Brain & AD Brain & WT Heart & AD Heart & WT Liver & AD Liver \\
\hline${ }^{\text {bBiological Replicate 1 }}$ & 2055 & 2065 & 2058 & 2062 & 2052 & 2040 \\
\hline${ }^{\text {cBiological Replicate 2 }}$ & 1662 & 1661 & 1654 & 1660 & 1661 & 1667 \\
\hline${ }^{\text {bBiological Replicate 3 }}$ & 2030 & 1975 & 2030 & 2017 & 2012 & 2015 \\
\hline${ }^{\text {CBiological Replicate 4 }}$ & 1692 & 1691 & 1696 & 1644 & 1700 & 1686 \\
\hline${ }^{\text {bBiological Replicate 5 }}$ & 2166 & 2162 & 2152 & 2146 & 2161 & 2152 \\
\hline${ }^{\text {cBiological Replicate 6 }}$ & 1752 & 1752 & 1744 & 1744 & 1752 & 1755 \\
\hline d'Average \pm SD & $1893 \pm 216$ & $1884 \pm 211$ & $1889 \pm 215$ & $1879 \pm 221$ & $1890 \pm 211$ & $1886 \pm 208$ \\
\hline
\end{tabular}

aThe number of proteins identified in each tissue/disease-state ( $N$ ranges between 1-6 biological replicates); WT Brain ( $N=3560)$, $A D$ Brain $(\mathrm{N}=3542)$, WT Heart $(\mathrm{N}=3569)$, AD Heart $(\mathrm{N}=3548)$, WT Liver $(\mathrm{N}=3567)$, and AD Liver $(\mathrm{N}=3559)$. $\mathrm{b}$ \& $\mathrm{c}$ The number of proteins quantified per biological replicate from $\mathrm{b}$ ) light- or c) heavy-dimethylated peptides, respectively. ${ }^{\mathrm{d}}$ The number of proteins (average \pm standard deviation) quantified in each tissue/disease-state. 
Supplemental Tables S8: Proteins quantified in one or two tissue types.

\begin{tabular}{|l|l|l|l|}
\hline Accession no & Protein description & Tissue(s) quantified & Highest level of expression \\
\hline E9QPX1 & Collagen alpha-1 (XVIII) chain & Heart & Aorta (Heart) \\
\hline E9Q842 & Neuron Navigator 2 & Brain and Heart & Heart \\
\hline O88444 & Adenylate cyclase type 1 & Brain and Heart & Cerebellum (Brain) \\
\hline Q8BYC4 & G-protein coupled receptor 20 & Brain and Heart & Carotid Artery (Heart) \\
\hline F7D6J5 & Sodium channel protein & Brain and Heart & Brain \\
\hline P52792 & Glucokinase & Liver & Liver \\
\hline
\end{tabular}


Supplemental Table S9: Differentially - expressed proteins in the brain, heart, and/or liver as a function of disease.

\begin{tabular}{|c|c|c|c|c|c|c|c|}
\hline Accession & Protein Description & AD/WT brain & $p$ value & AD/WT heart & p value & AD/WT liver & p value \\
\hline P08226 & Apolipoprotein E & $2.10 \pm 0.38$ & $6.14 \mathrm{E}-06$ & $1.02 \pm 0.12$ & $9.10 \mathrm{E}-01$ & $1.13 \pm 0.079$ & $6.80 \mathrm{E}-02$ \\
\hline P18242 & Cathepsin D & $1.24 \pm 0.093$ & $9.72 \mathrm{E}-04$ & $1.01 \pm 0.086$ & 7.90E-01 & $0.99 \pm 0.097$ & 9.10E-01 \\
\hline E9PWX1 & Dipeptidyl aminopeptidase-like protein 6 & $0.85 \pm 0.1$ & $1.20 \mathrm{E}-03$ & - & - & - & - \\
\hline Q01853 & Transitional endoplasmic reticulum ATPase & $1.05 \pm 0.033$ & $1.97 \mathrm{E}-03$ & $1.04 \pm 0.09$ & $3.00 \mathrm{E}-01$ & $1.04 \pm 0.073$ & 1.10E-01 \\
\hline 008599 & Syntaxin-binding protein 1 & $0.87 \pm 0.11$ & $3.29 \mathrm{E}-03$ & - & - & - & - \\
\hline E9QOH6 & Fatty acid-binding protein, brain & $1.46 \pm 0.61$ & $5.44 \mathrm{E}-03$ & $1.02 \pm 0.26$ & 8.70E-01 & $0.98 \pm 0.049$ & 8.40E-01 \\
\hline Q91ZA3 & Propionyl-CoA carboxylase alpha chain, mitochondrial & $1.06 \pm 0.051$ & $9.29 \mathrm{E}-03$ & $1.00 \pm 0.074$ & $9.60 \mathrm{E}-01$ & $0.97 \pm 0.033$ & 4.00E-01 \\
\hline P63040 & Complexin-1 & $1.08 \pm 0.089$ & 1.32E-02 & - & - & - & - \\
\hline Q9CPT4 & Myeloid-derived growth factor & $1.18 \pm 0.12$ & 1.42E-02 & - & - & $0.93 \pm 0.045$ & 8.30E-02 \\
\hline Q9D0M5 & Dynein light chain 2, cytoplasmic & $1.10 \pm 0.081$ & 1.59E-02 & $1.07 \pm 0.14$ & $2.80 \mathrm{E}-01$ & $1.04 \pm 0.11$ & 7.00E-01 \\
\hline Q9CQU0 & Thioredoxin domain-containing protein 12 & $1.17 \pm 0.063$ & 1.77E-02 & - & - & $0.95 \pm 0.11$ & $5.80 \mathrm{E}-01$ \\
\hline Q06890 & Clusterin & $1.61 \pm 0.81$ & $1.79 \mathrm{E}-02$ & $0.92 \pm 0.20$ & $3.60 \mathrm{E}-01$ & $0.93 \pm 0.075$ & 4.40E-01 \\
\hline Q9CQ16 & Coactosin-like protein & $1.28 \pm 0.37$ & 1.95E-02 & $0.90 \pm 0.13$ & - & $1.08 \pm 0.17$ & 4.30E-01 \\
\hline P27773 & Protein disulfide-isomerase $\mathrm{A} 3$ & $1.10 \pm 0.066$ & 2.09E-02 & $1.04 \pm 0.11$ & 7.70E-01 & $0.99 \pm 0.046$ & 6.40E-01 \\
\hline Q9Z1Q5 & Chloride intracellular channel protein 1 & $1.29 \pm 0.34$ & 2.10E-02 & $1.05 \pm 0.069$ & $5.50 \mathrm{E}-01$ & $1.02 \pm 0.087$ & 8.20E-01 \\
\hline P14211 & Calreticulin & $1.11 \pm 0.027$ & 2.14E-02 & $1.06 \pm 0.11$ & 4.70E-01 & $0.96 \pm 0.046$ & $1.90 \mathrm{E}-01$ \\
\hline P63318 & Protein kinase $\mathrm{C}$ gamma type & $0.84 \pm 0.13$ & 2.14E-02 & - & - & - & - \\
\hline Q9CYRO & Single-stranded DNA-binding protein, mitochondrial & $1.16 \pm 0.086$ & $2.28 \mathrm{E}-02$ & $1.02 \pm 0.22$ & 8.00E-01 & $0.97 \pm 0.091$ & $6.60 \mathrm{E}-01$ \\
\hline P57759 & Endoplasmic reticulum resident protein 29 & $1.32 \pm 0.47$ & 2.32E-02 & $1.08 \pm 0.054$ & $4.50 \mathrm{E}-01$ & $0.98 \pm 0.11$ & $7.20 \mathrm{E}-01$ \\
\hline P11438 & Lysosome-associated membrane glycoprotein 1 & $1.19 \pm 0.12$ & 2.41E-02 & $1.04 \pm 0.18$ & $6.80 \mathrm{E}-01$ & $0.98 \pm 0.052$ & 5.00E-01 \\
\hline Q9CRD0 & OCIA domain-containing protein 1 & $0.73 \pm 0.23$ & 2.62E-02 & $0.98 \pm 0.40$ & - & $0.73 \pm 0.20$ & 9.70E-02 \\
\hline Q99LB2 & Dehydrogenase/reductase SDR family member 4 & $1.20 \pm 0.24$ & 2.77E-02 & $1.01 \pm 0.095$ & 7.90E-01 & $1.05 \pm 0.099$ & 9.70E-02 \\
\hline Q8BFZ9 & Erlin-2 & $1.20 \pm 0.23$ & 3.12E-02 & $1.03 \pm 0.19$ & $8.00 \mathrm{E}-01$ & $1.05 \pm 0.047$ & $9.20 \mathrm{E}-02$ \\
\hline AOAOJ9YUN4 & Dynamin-1 & $0.84 \pm 0.19$ & 3.34E-02 & - & - & - & - \\
\hline Q9WV54 & Acid ceramidase & $1.17 \pm 0.17$ & 3.43E-02 & $1.13 \pm 0.24$ & $4.00 \mathrm{E}-01$ & $0.96 \pm 0.10$ & 5.40E-01 \\
\hline Q6GT24 & Peroxiredoxin-6 & $1.14 \pm 0.11$ & 3.43E-02 & $0.92 \pm 0.071$ & $4.80 \mathrm{E}-01$ & $1.05 \pm 0.11$ & 3.00E-01 \\
\hline Q9D6F9 & Tubulin beta- $4 A$ chain & $0.95 \pm 0.048$ & 3.68E-02 & - & - & - & - \\
\hline P35700 & Peroxiredoxin-1 & $1.14 \pm 0.10$ & 3.80E-02 & $1.05 \pm 0.073$ & $3.70 \mathrm{E}-01$ & $1.05 \pm 0.061$ & 4.84E-02 \\
\hline P07901 & Heat shock protein HSP 90-alpha & $1.06 \pm 0.052$ & 3.84E-02 & $0.98 \pm 0.085$ & 8.30E-01 & $0.96 \pm 0.037$ & 6.30E-01 \\
\hline Q5SXR6 & Clathrin heavy chain & $0.87 \pm 0.18$ & 4.17E-02 & $1.00 \pm 0.17$ & $9.70 \mathrm{E}-01$ & $1.03 \pm 0.063$ & 5.40E-01 \\
\hline Q3UJU9 & Regulator of microtubule dynamics protein 3 & $1.38 \pm 0.49$ & 4.27E-02 & - & - & $1.04 \pm 0.037$ & 4.30E-01 \\
\hline Q8BFR5 & Elongation factor Tu, mitochondrial & $1.09 \pm 0.10$ & 4.30E-02 & $1.01 \pm 0.039$ & $8.40 \mathrm{E}-01$ & $1.00 \pm 0.039$ & 5.30E-02 \\
\hline P08113 & Endoplasmin & $1.12 \pm 0.47$ & 4.33E-02 & $1.03 \pm 0.097$ & 7.30E-01 & $0.96 \pm 0.064$ & 1.10E-01 \\
\hline Q9DAW9 & Calponin-3 & $1.26 \pm 0.43$ & 4.43E-02 & $1.20 \pm 0.27$ & - & $1.06 \pm 0.39$ & 5.90E-01 \\
\hline Q8R4NO & Citramalyl-CoA lyase, mitochondrial & $1.09 \pm 0.13$ & 4.43E-02 & $1.02 \pm 0.10$ & $6.80 \mathrm{E}-01$ & $1.00 \pm 0.054$ & $9.90 \mathrm{E}-01$ \\
\hline P01831 & Thy-1 membrane glycoprotein & $0.68 \pm 0.39$ & 4.59E-02 & $0.96 \pm 0.069$ & - & $1.02 \pm 0.083$ & - \\
\hline Q9D019 & Arginine--tRNA ligase, cytoplasmic & $1.16 \pm 0.11$ & 4.60E-02 & $1.07 \pm 0.32$ & $5.90 \mathrm{E}-01$ & $1.04 \pm 0.11$ & 3.50E-01 \\
\hline Q8KOTO & Reticulon-1 & $0.88 \pm 0.14$ & 4.72E-02 & $1.00 \pm 0.14$ & $9.60 \mathrm{E}-01$ & - & - \\
\hline P35980 & $60 S$ ribosomal protein $\mathrm{L} 18$ & $0.75 \pm 0.19$ & 4.82E-02 & $1.04 \pm 0.20$ & $8.60 \mathrm{E}-01$ & $0.82 \pm 0.24$ & $1.20 \mathrm{E}-01$ \\
\hline Q99N87 & $28 S$ ribosomal protein $\mathrm{S} 5$, mitochondrial & $0.80 \pm 0.16$ & 7.32E-02 & $1.21 \pm 0.24$ & 2.31E-02 & $1.08 \pm 0.12$ & 2.60E-01 \\
\hline Q792Y8 & MCG15081 & $1.08 \pm 0.13$ & 7.70E-02 & $1.07 \pm 0.16$ & $2.90 \mathrm{E}-01$ & $1.20 \pm 0.089$ & 9.67E-05 \\
\hline P26043 & Radixin & $1.11 \pm 0.16$ & $8.30 \mathrm{E}-02$ & $0.99 \pm 0.12$ & 8.50E-01 & $1.14 \pm 0.10$ & 5.36E-03 \\
\hline 008715 & A-kinase anchor protein 1 , mitochondrial & $1.24 \pm 0.32$ & $1.36 \mathrm{E}-01$ & $0.88 \pm 0.14$ & 8.90E-02 & $1.22 \pm 0.084$ & 3.59E-04 \\
\hline Q920E5 & Farnesyl pyrophosphate synthase & $1.20 \pm 0.23$ & 2.10E-01 & $0.99 \pm 0.22$ & - & $1.28 \pm 0.53$ & 3.66E-02 \\
\hline Q8BTY1 & Kynurenine--oxoglutarate transaminase 1 & $1.15 \pm 0.34$ & $2.20 \mathrm{E}-01$ & $1.13 \pm 0.37$ & 4.60E-01 & $0.89 \pm 0.052$ & 2.29E-02 \\
\hline Q9CZ13 & Cytochrome b-c1 complex subunit 1 , mitochondrial & $1.06 \pm 0.16$ & $2.80 \mathrm{E}-01$ & $1.13 \pm 0.12$ & $9.38 E-03$ & $0.96 \pm 0.054$ & 4.10E-01 \\
\hline O88844 & Isocitrate dehydrogenase [NADP] cytoplasmic & $1.10 \pm 0.052$ & $3.00 \mathrm{E}-01$ & $1.02 \pm 0.069$ & $7.80 \mathrm{E}-01$ & $1.10 \pm 0.067$ & $1.48 \mathrm{E}-03$ \\
\hline Q8VCC2 & Liver carboxylesterase 1 & $1.17 \pm 0.44$ & $3.20 \mathrm{E}-01$ & - & - & $1.12 \pm 0.18$ & 2.48E-02 \\
\hline Q6PA06 & Atlastin-2 & $1.08 \pm 0.25$ & 3.90E-01 & - & - & $1.14 \pm 0.18$ & 4.96E-02 \\
\hline P06801 & NADP-dependent malic enzyme & $1.10 \pm 0.21$ & 4.10E-01 & $1.07 \pm 0.18$ & 3.90E-01 & $1.42 \pm 0.31$ & $1.28 \mathrm{E}-03$ \\
\hline P42125 & Enoyl-CoA delta isomerase 1 , mitochondrial & $1.14 \pm 0.36$ & 4.40E-01 & $0.99 \pm 0.11$ & $2.10 \mathrm{E}-01$ & $1.06 \pm 0.035$ & 1.12E-02 \\
\hline B1ATIO & Aldehyde dehydrogenase & $1.07 \pm 0.11$ & 4.60E-01 & $1.02 \pm 0.27$ & - & $1.22 \pm 0.24$ & $1.89 \mathrm{E}-02$ \\
\hline P23953 & Carboxylesterase 1C & $1.09 \pm 0.27$ & $5.00 \mathrm{E}-01$ & $1.00 \pm 0.098$ & $9.40 \mathrm{E}-01$ & $1.16 \pm 0.054$ & 1.81E-02 \\
\hline Q99MN9 & Propionyl-CoA carboxylase beta chain, mitochondrial & $1.05 \pm 0.20$ & $5.70 \mathrm{E}-01$ & $1.10 \pm 0.062$ & 1.83E-02 & $0.95 \pm 0.018$ & $1.50 \mathrm{E}-01$ \\
\hline P97855 & Ras GTPase-activating protein-binding protein 1 & $0.94 \pm 0.22$ & $5.70 \mathrm{E}-01$ & $0.84 \pm 0.10$ & 2.68E-02 & $1.00 \pm 0.16$ & $1.00 \mathrm{E}+00$ \\
\hline AOAOAOMQF6 & Glyceraldehyde-3-phosphate dehydrogenase & $0.99 \pm 0.044$ & $5.80 \mathrm{E}-01$ & $0.94 \pm 0.037$ & 1.73E-02 & $0.95 \pm 0.074$ & 8.30E-01 \\
\hline P24549 & Retinal dehydrogenase 1 & $1.07 \pm 0.23$ & $6.00 \mathrm{E}-01$ & $1.05 \pm 0.17$ & 7.70E-01 & $1.14 \pm 0.21$ & 2.64E-02 \\
\hline Q9ROHO & Peroxisomal acyl-coenzyme A oxidase 1 & $1.08 \pm 0.19$ & $6.20 \mathrm{E}-01$ & $1.02 \pm 0.16$ & $8.00 \mathrm{E}-01$ & $1.14 \pm 0.095$ & 3.16E-04 \\
\hline Q8BG32 & 265 proteasome non-ATPase regulatory subunit 11 & $0.97 \pm 0.19$ & 7.10E-01 & $1.25 \pm 0.40$ & 4.88E-02 & $0.97 \pm 0.074$ & 4.40E-01 \\
\hline Q9JII6 & Alcohol dehydrogenase $[\mathrm{NADP}(+)]$ & $1.01 \pm 0.037$ & 7.70E-01 & - & - & $1.12 \pm 0.15$ & 4.31E-02 \\
\hline Q9QYJ0 & $\begin{array}{l}\text { DnaJ homolog subfamily A member } 2 \\
\text { NADH dehydrogenase [ubiquinone] } 1 \text { alpha }\end{array}$ & $1.01 \pm 0.12$ & 8.40E-01 & $0.91 \pm 0.10$ & 4.87E-02 & $1.08 \pm 0.097$ & 2.40E-01 \\
\hline Q9DC69 & subcomplex subunit 9 , mitochondrial & $0.99 \pm 0.14$ & 8.70E-01 & $0.90 \pm 0.14$ & 4.91E-02 & $1.02 \pm 0.097$ & 8.00E-01 \\
\hline P09103 & $\begin{array}{l}\text { Protein disulfide-isomerase } \\
\text { NADH dehydrogenase [ubiquinone] } 1 \text { alpha }\end{array}$ & $1.01 \pm 0.088$ & 8.80E-01 & $1.03 \pm 0.25$ & 8.40E-01 & $0.94 \pm 0.064$ & 4.36E-02 \\
\hline Q9ERS2 & subcomplex subunit 13 & $1.01 \pm 0.090$ & $8.90 \mathrm{E}-01$ & $1.10 \pm 0.029$ & 4.62E-02 & $0.97 \pm 0.097$ & 8.60E-01 \\
\hline Q8QZT1 & Acetyl-CoA acetyltransferase, mitochondrial & $1.01 \pm 0.098$ & $9.00 \mathrm{E}-01$ & $0.99 \pm 0.091$ & $7.20 \mathrm{E}-01$ & $1.11 \pm 0.088$ & 2.57E-02 \\
\hline Q9D0M3 & Cytochrome $\mathrm{c} 1$, heme protein, mitochondrial & $1.01 \pm 0.11$ & $9.20 \mathrm{E}-01$ & $1.10 \pm 0.11$ & 3.89E-02 & $0.93 \pm 0.073$ & 3.70E-01 \\
\hline Q921H8 & 3-ketoacyl-CoA thiolase A, peroxisomal & $1.00 \pm 0.13$ & $9.60 \mathrm{E}-01$ & $1.02 \pm 0.20$ & $9.00 \mathrm{E}-01$ & $1.15 \pm 0.18$ & 2.62E-02 \\
\hline Q9DCZ4 & MICOS complex subunit Mic26 & $0.99 \pm 0.067$ & $9.90 \mathrm{E}-01$ & $1.07 \pm 0.078$ & 4.57E-02 & $0.94 \pm 0.11$ & 6.40E-01 \\
\hline P06330 & Ig heavy chain $\vee$ region $A C 38205.12$ & $1.24 \pm 0.57$ & - & $0.63 \pm 0.18$ & 1.66E-02 & $0.70 \pm 0.20$ & 4.46E-02 \\
\hline P16015 & Carbonic anhydrase 3 & $1.07 \pm 0.20$ & - & $1.01 \pm 0.17$ & $9.40 \mathrm{E}-01$ & $1.28 \pm 0.27$ & $7.80 E-04$ \\
\hline Q71KT5 & Delta(14)-sterol reductase & $0.99 \pm 0.22$ & - & $1.09 \pm 0.22$ & - & $1.20 \pm 0.27$ & 3.65E-02 \\
\hline Q8VCHO & 3-ketoacyl-CoA thiolase B, peroxisomal & $1.05 \pm 0.18$ & - & $0.99 \pm 0.13$ & $9.40 \mathrm{E}-01$ & $1.35 \pm 0.26$ & 2.80E-04 \\
\hline Q91X83 & $\mathrm{S}$-adenosylmethionine synthase isoform type-1 & $0.92 \pm 0.25$ & - & $1.20 \pm 0.27$ & - & $1.23 \pm 0.35$ & $1.25 \mathrm{E}-02$ \\
\hline Q9DCT8 & Cysteine-rich protein 2 & - & - & $1.08 \pm 0.039$ & 3.34E-02 & - & - \\
\hline A0A0R4J083 & mitochondrial & - & - & $1.04 \pm 0.033$ & 4.64E-02 & $1.04 \pm 0.051$ & $9.70 \mathrm{E}-01$ \\
\hline 070310 & Glycylpeptide $\mathrm{N}$-tetradecanoyltransferase 1 & - & - & - & - & $1.20 \pm 0.21$ & 2.19E-02 \\
\hline P24270 & Catalase & - & - & - & - & $1.08 \pm 0.086$ & 4.20E-02 \\
\hline P33267 & Cytochrome P450 2F2 & - & - & - & - & $0.85 \pm 0.090$ & 9.48E-04 \\
\hline P50431 & Serine hydroxymethyltransferase, cytosolic & - & - & - & - & $1.15 \pm 0.099$ & $1.22 \mathrm{E}-02$ \\
\hline Q5FW57 & Glycine $\mathrm{N}$-acyltransferase-like protein & - & - & - & - & $1.12 \pm 0.099$ & $6.23 \mathrm{E}-03$ \\
\hline Q8VCT4 & Carboxylesterase 1D & - & - & $1.07 \pm 0.16$ & 7.10E-01 & $1.13 \pm 0.10$ & 2.35E-03 \\
\hline Q8VCU1 & Carboxylesterase 3B & - & - & - & - & $0.89 \pm 0.069$ & $8.82 \mathrm{E}-03$ \\
\hline Q9DBE0 & Cysteine sulfinic acid decarboxylase & - & - & - & - & $1.70 \pm 1.07$ & $9.91 \mathrm{E}-03$ \\
\hline
\end{tabular}

